\title{
Chaotic gas bubble oscillations in a viscoelastic fluid
}

\author{
Javier Jiménez-Fernández \\ Dpto. Ingeniería Energética y Fluidomecánica, E.T.S.I. Industriales, 28006 Madrid, Spain
}

\begin{abstract}
Regular and chaotic radial oscillations of an acoustically driven gas bubble in a viscoelastic fluid have been theoretically analyzed. For parameter values usually found in diagnostic ultrasound period-doubling routes to chaos have been identified. Thresholds values of the external pressure amplitude for a first bifurcation in terms of the elasticity and the shear viscosity of the host fluid have also been evaluated.
\end{abstract}

\section{Résumé}

Oscillations chaotiques d'une bulle de gaz au sein d'un fluide viscoélastique. Les oscillations radiales, régulières ou chaotiques, d'une bulle de gaz au sein d'un fluide viscoélastique et soumise à l'action d'un champ acoustique sont analysées théoriquement. Pour des valeurs des paramètres qui sont normalement rencontrées dans le diagnostic médical, des chemins vers le chaos par doublement de la période ont été identifiés. Les valeurs limites pour la première bifurcation en fonction de l'élasticité et de la viscosité du fluide, ont été aussi déterminées.

Keywords: Acoustics; Bubble dynamics; Ultrasound; Viscoelastic fluids

Mots-clés : Acoustique; Dynamique des bulles; Ultrasons ; Fluides viscoelastiques

\section{Introduction}

A gas bubble immersed in a liquid and subjected to the action of an external acoustical field behaves like a nonlinear oscillator. Thus, depending on the values of the frequency and of the external pressure amplitude the bubble undergoes radial oscillations which may be steady or chaotic Besides basic aspects, the non-linear dynamics of gas bubbles is a subject of interest in numerous areas and particularly in the biomedical field where gas bubbles as well as encapsulated gas bubbles, are proven to be very valuable tools as contrast agents for medical ultrasound diagnosis. More recently, the use of microbubbles for non-invasive therapy and drug delivery as well as in high intensity focused ultrasound treatments has also been recognized as an efficient tool in clinical applications 
For frequencies and bubble equilibrium radii usually found in diagnostic applications (1-10 MHz, 1-5 $\mu \mathrm{m})$ chaotic behaviors could be expected for moderate values of the external pressure amplitude when the host fluid is water. For instance, for a free gas bubble of $1 \mu \mathrm{m}$ driven at $2.5 \mathrm{MHz}$ in water, a first bifurcation occurs for an amplitude of $160 \mathrm{kPa}$ and chaos for an amplitude of about $280 \mathrm{kPa}$. However, if the viscosity is increased to $30 \mathrm{mPa}$ s, a gas bubble with the same radius and for the same frequency undergoes a first bifurcation for $p_{A}=3.7 \mathrm{MPa}$ and a chaotic oscillation for $p_{A}=3.9 \mathrm{MPa}$, i.e., for pressure amplitudes considerably greater. So, taking into account that the viscosity values of biological fluids or biological tissues may be significant larger than the viscosity of water $(\sim 5 \mathrm{cP}$ for blood, $\sim 50 \mathrm{cP}$ for the synovial fluid) it should be expected that the radial bubble oscillations encountered in diagnostic ultrasound applications be periodic. Nevertheless, it is well recognized that biological fluids exhibit a Non-Newtonian behavior and hence, besides viscosity, other physical parameters must be considered in order to provide a realistic description of bubble dynamics in living tissues In this context, recent works on the dynamics of gas bubbles immersed in viscoelastic fluids have shown that fluid elasticity enhances the radial oscillations so that even for moderate values of the external amplitude the behavior may be chaotic In fact, as it will be shown below, fluid elasticity may reduce dramatically the threshold values of the driven pressure amplitudes for bifurcation and chaos.

In this work, the dynamics of spherical gas bubbles in viscoelastic fluids subjected to an external acoustic field is studied. The problem is formulated considering for the extra stress tensor a differential constitutive equation with an interpolated time derivative: a form of the 4-constant Oldroyd model. It is confirmed that fluid elasticity produces a significant growth of the amplitude of bubble oscillations. Furthermore, it is found that as the Deborah number (the ratio between the relaxation time of the fluid and the characteristic time of the flow) is increased, the radial oscillations undergo period-doubling bifurcations and become chaotic. Finally, the threshold values of the driven pressure amplitude for which the first bifurcation occurs have been determined in terms of both the fluid elasticity as well as the shear viscosity. These values may provide useful information for diagnostic applications about the region of the parameter space where periodic or chaotic oscillations could be expected.

\section{Governing equations}

Consider a spherical bubble with an equilibrium radius $R_{0}$ immersed in a viscoelastic fluid. The dynamics of the bubble is governed by the Rayleigh-Plesset equation which in a spherical coordinate system centered at the bubble center, may be written in the form [3]:

$$
\rho\left(R \ddot{R}+\frac{3}{2} \dot{R}^{2}\right)=p_{g}-p_{\infty}-\frac{2 \sigma}{R}+2 \int_{R}^{\infty} \frac{\tau_{r r}-\tau_{\theta \theta}}{r} \mathrm{~d} r
$$

where $R$ is the instantaneous bubble radius and the dot denotes differentiation with time, $\rho$ is the liquid density and $\sigma$ is the coefficient of surface tension. $p_{\infty}=p_{0}+p_{A} \sin (2 \pi f t)$, is the driving sound field, where $p_{0}$ is the ambient pressure, $p_{A}$ is the driving pressure amplitude and $f$ is the acoustic frequency. $p_{g}$ is the uniform gas pressure inside the bubble which is assumed to be given by an adiabatic relation: $p_{g}=p_{g 0}\left(R_{0} / R\right)^{3 \gamma}$, where $\gamma=1.4$ and $p_{g 0}$ and $R_{0}$ are respectively, the gas bubble pressure and the bubble radius at the initial equilibrium state, that is: $p_{g 0}=p_{0}+2 \sigma / R_{0}$. Finally, $\tau_{r r}$ and $\tau_{\theta \theta}$ are the components of the extra stress tensor $\tau$ which must be defined by means of an appropriate constitutive equation. Radial bubble motions are elongational flows which, according to previous analyses [3,5], are adequately described by the following constitutive equation:

$$
\begin{aligned}
& \boldsymbol{\tau}=\boldsymbol{\tau}^{p}+2 \eta_{s} \mathbf{e} \\
& \boldsymbol{\tau}^{p}+\lambda\left(\frac{D \boldsymbol{\tau}^{p}}{D t}-\frac{1}{2}\left(\boldsymbol{\tau}^{p} \mathbf{e}+\mathbf{e} \boldsymbol{\tau}^{p}\right)\right)=2 \eta_{p} \mathbf{e}
\end{aligned}
$$

In the above expressions, $\eta_{s}$ and $\eta_{p}$ are shear viscosity coefficients so that $\eta=\eta_{s}+\eta_{p}$, is the shear viscosity of the host fluid, and $\lambda$ is a stress relaxation time. $\mathbf{e}$ is the rate of strain tensor defined as: $\mathbf{e}=\frac{1}{2}\left(\nabla \mathbf{v}+(\nabla \mathbf{v})^{T}\right)$ where $\nabla \mathbf{v}$ is the velocity gradient, and $\frac{D \boldsymbol{\tau}^{p}}{D t}=\frac{\partial \tau^{p}}{\partial t}+\mathbf{v} \nabla \boldsymbol{\tau}^{p}$ is the usual convective derivative. As it may be noted, mass diffusion and compressibility effects have been neglected in the present formulation. These are quantitatively important effects but here interest is mainly focused in studying the role played by the fluid elasticity in order to compare the Newtonian behavior versus the viscoelastic behavior. 
After a transformation to Lagrangian coordinates Eqs. (1)-(3) may be reduced to the differential system:

$$
\begin{aligned}
& \rho\left(R \ddot{R}+\frac{3}{2} \dot{R}^{2}\right)=p_{g}-p_{\infty}-\frac{2 \sigma}{R}-4 \eta_{s} \frac{\dot{R}}{R}+S_{p} \\
& \dot{S}_{p}=-\left(\frac{1}{\lambda}+2 \frac{\dot{R}}{R}\right) S_{p}-4 \frac{\eta_{p}}{\lambda} \frac{\dot{R}}{R}
\end{aligned}
$$

where:

$$
S_{p}(t)=2 \int_{R}^{\infty} \frac{\tau_{r r}^{p}(t)-\tau_{\theta \theta}^{p}(t)}{r} \mathrm{~d} r
$$

\section{Numerical results}

\subsection{Steady oscillations and transition to chaos}

First, we analyze the influence of the fluid elasticity on the bubble radius evolution in time. To this end, the system (4), (5) has been integrated with the initial conditions: $R(0)=R_{0}, \dot{R}(0)=S_{p}(0)=0$. Numerical calculations have been performed with Mathematica (Wolfram Research Inc.). As usual, the fluid elasticity is here quantified by means of the Deborah number defined by: $D e=\lambda / t_{c}$, i.e., the ratio between the relaxation time of the fluid $\lambda$, and the characteristic time of the flow given by $t_{c}=R_{0} \sqrt{\rho / p_{0}}$. Normalized bubble radius $R^{*}=R / R_{0}$ versus time (acoustic periods) curves and Poincaré section plots: $V_{p}^{*}=\dot{R}_{p} t_{c} / R_{0}$ versus $R_{p}^{*}=R_{p} / R_{0}$ curves, where $R_{p}$ is the bubble radius at the end of each cycle, are shown in Fig. 1 for a system of interest in ultrasound medical applications [4]: $R_{0}=1 \mu \mathrm{m}, \rho=10^{3} \mathrm{~kg} / \mathrm{m}^{3}, \sigma=0.072 \mathrm{~N} / \mathrm{m}$, and $\eta=30 \mathrm{cP}$, for a pressure amplitude $p_{A}=1.5 \mathrm{MPa}$, and an acoustic frequency $f=3 \mathrm{MHz}$. Four values of the Deborah number have been considered: $D e=0$ (Newtonian case) $D e=0.25, D e=0.5$, and $D e=1.25$ respectively. As it may be observed, in a Newtonian fluid (1st row) the bubble oscillates with the period of the driving acoustic field. For a viscoelastic host fluid with $D e=0.25$ ( 2 nd row) a period-2 oscillation occurs. For $D e=0.5$ (3rd row) the period is doubled again, and finally for $D e=1.25$ (4th row), the behavior becomes chaotic. This transition to chaos as the Deborah number is increased may be more clearly visualized by means of bifurcation diagrams as the one shown in Fig. 2, where the normalized bubble radius $R_{p} / R_{0}$ at a given phase of the driving sound field is plotted versus the Deborah number which is taken as control parameter. This diagram has been performed for the system considered in Fig. 1 for an external amplitude $p_{A}=1.5 \mathrm{MPa}$ and an acoustic frequency $f=3 \mathrm{MHz}$. For any value of the Deborah number, starting with $D e=0.01$, the differential system (4), (5) has been solved for 500 acoustic cycles and the values of $R$ at the end of each cycle have been plotted (dropping the first 300 values). This diagram shows indeed a period-doubling bifurcation route to chaos as the Deborah number increases.

\subsection{Thresholds for the first bifurcation}

We have seen in the previous section that a gas bubble which undergoes stable radial oscillations in a Newtonian fluid may have a chaotic behaviour in a viscoelastic fluid. In other words, for fixed $D e$ there is a threshold value of the external pressure amplitude below which the oscillations are periodic. From a practical point of view it is of interest to determine this value in order to gain a first insight on the parameter space region where regular or chaotic oscillations may be expected. This threshold value is drastically reduced as the De number increases, as it is shown in Fig. 3, where results are plotted for the system of Fig. 1 and the frequencies $f=1,3,5 \mathrm{MHz}$, respectively.

Finally, the quantitative influence of the dynamic viscosity on the threshold pressure values for a first bifurcation is also evaluated. As it is shown in Fig. 4, for the system of Fig. 1 linear behaviors are observed in the Newtonian case as well as in the viscoelastic case for $D e=1$ in the interval considered: $1-30 \mathrm{mPa}$. It must be remarked, however, that the slope for $D e=1$ is clearly smaller than the slope for a Newtonian fluid. Thus, the influence of the fluid elasticity on the thresholds values for bifurcation becomes increasingly important as the shear viscosity is raised.

It may be concluded, therefore, that fluid elasticity cancels out viscous damping so that in a viscoelastic fluid the threshold values for bifurcation and chaos are largely reduced. The above results suggest that further work about 

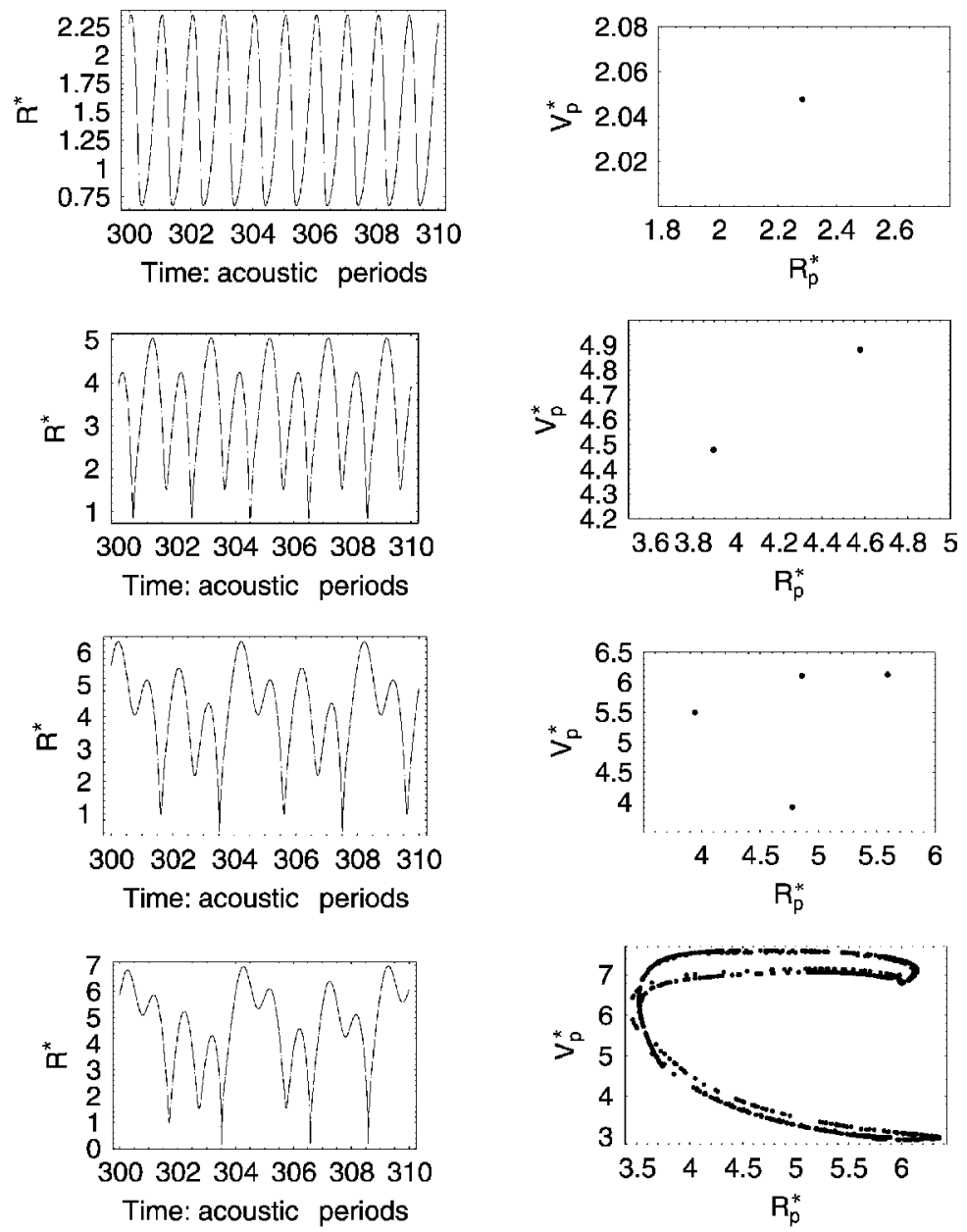

Fig. 1. Left column: Normalized bubble radius $R^{*}$ versus time in acoustic periods. Right column: Poincaré section plots: $V_{p}^{*}$ versus $R_{p}^{*}$. $R_{0}=1 \mu m$, $\rho=10^{3} \mathrm{Kg} / \mathrm{m}^{3}, \sigma=0.072 \mathrm{~N} / \mathrm{m}, \eta=30 \mathrm{cP}, \varepsilon=0.2, f=3 \mathrm{MHz}, p_{A}=1.5 \mathrm{MPa}$. 1st row: $D e=0$ (Newtonian fluid), 2nd row $D e=0.25,3 \mathrm{rd}$ row: $D e=0.5$, 4th row: $D e=1.25$.

Fig. 1. Colonne de gauche : Rayon de la bubble normalisé $R^{*}$ en function du temps en périodes acoustiques. Colonne de droite : sections de Poincaré : $V_{p}^{*}$ versus $R_{p}^{*} . R_{0}=1 \mu \mathrm{m}, \rho=10^{3} \mathrm{Kg} / \mathrm{m}^{3}, \sigma=0.072 \mathrm{~N} / \mathrm{m}, \eta=30 \mathrm{cP}, \varepsilon=0.2, f=3 \mathrm{MHz}, p_{A}=1.5 \mathrm{MPa}$. 1 ère ligne $: D e=0$ (fluide newtonien), 2ème ligne $D e=0.25$, 3ème ligne : $D e=0.5$, 4ème ligne : $D e=1.25$.

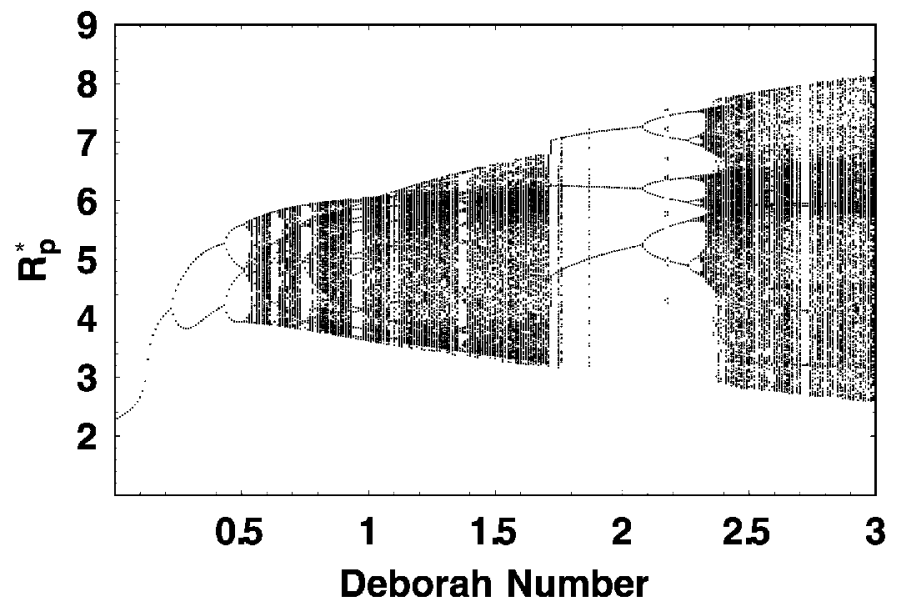

Fig. 2. Bifurcation diagram. Control parameter: Deborah number. Data as in Fig. $1, f=3 \mathrm{MHz}, p_{A}=1.5 \mathrm{MPa}$.

Fig. 2. Diagramme de bifurcation. Paramètre de contrôle : Nombre de Deborah. Données de la Fig. $1 f=3 \mathrm{MHz}, p_{A}=1.5 \mathrm{MPa}$. 


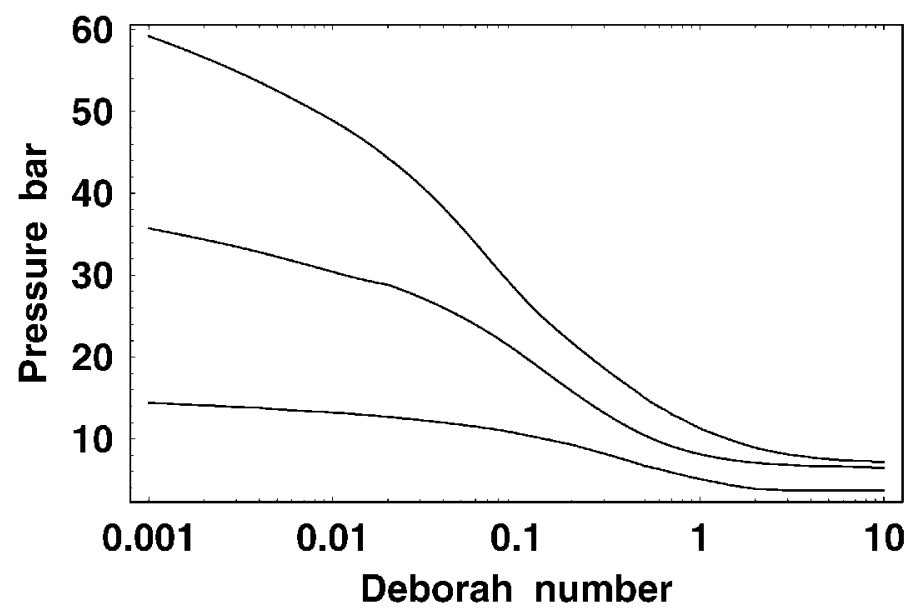

Fig. 3. Pressure amplitude threshold for the first bifurcation as function of the Deborah number. Data as in Fig. $1, f=1,3,5 \mathrm{MHz}$ (from bottom to top).

Fig. 3. Seuil de l'amplitude de la pression pour une première bifurcation en fonction du nombre de Deborah. Données de la Fig. 1, $f=1,3,5 \mathrm{MHz}$.

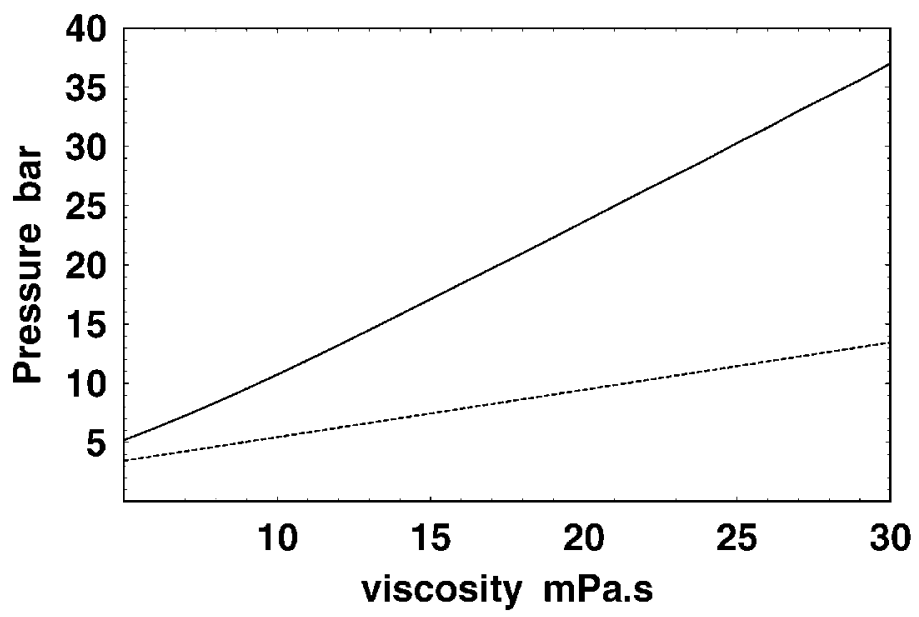

Fig. 4. Pressure amplitude threshold for the first bifurcation as function of the shear viscosity for a Newtonian fluid (dark line) and a viscoelastic fluid with $D e=1$ (dashed line). Data as in Fig. $1, f=3 \mathrm{MHz}$.

Fig. 4. Amplitude de la pression pour une première bifurcation en fonction de la viscosité pour un fluide Newtonien (ligne continue) et un fluide viscoélastique avec $D e=1$ (ligne pointillée). Données de la Fig. $1, f=3 \mathrm{MHz}$.

non-linear bubble dynamics in viscoelastic media should be done in order to provide a realistic description of bubble behavior in biological media.

\section{Acknowledgements}

The author wishes to acknowledge the financial support of the Dirección General de Investigación. Ministerio de Ciencia y Tecnología (Spain). Project: DPI2003-05643.

\section{References}

W. Lauterborn, U. Parlitz, Methods of chaos physics and their application to acoustics, J. Acoust. Soc. Am. 84 (6) (1988) $1975-1993$.

B.B. Goldberg, J.S. Raichlen, F. Fosberg (Eds.), Ultrasound Contrast Agents: Basic Principles and Clinical Applications, second ed., Martin Dunitz, London, 2001.

S.P. Levitskii, Z.P. Shulman, Bubbles in Polymeric Liquids: Dynamics, Heat and Mass Transfer, Technomic Publishing A G, Bassel, Switzerland, 1995. 
J.S. Allen, R.A. Roy, Dynamics of gas bubbles in viscoelastic fluids II. Nonlinear viscoelasticity, J. Acoust. Soc. Am. 108 (4) (2000) 16401650.

J. Jiménez-Fernández, A. Crespo, Bubble oscillation and inertial cavitation in viscoelastic fluids, U1trasonics 43 (2005) $643-651$.

J. Jiménez-Fernández, A. Crespo, The collapse of gas bubbles and cavities in a viscoelastic fluid, Int. J. Multiphase Flow 32 (2006) $1294-1299$. 